\title{
Prenatal Diagnosis of Fetal Ventriculomegaly: Agreement between Fetal Brain Ultrasonography and MR Imaging
}

\author{
S. Perlman, D. Shashar, C. Hoffmann, O.B. Yosef, R. Achiron, and E. Katorza
}

\begin{abstract}
BACKGROUND AND PURPOSE: Accurate measurement of the lateral ventricles is of paramount importance in prenatal diagnosis. Possible conflicting classifications caused by their measurement in different sectional planes by sonography and MR imaging are frequently raised. The objective of our study was to evaluate the agreement between ultrasonography and MR imaging in the measurement of the lateral ventricle diameter in the customary sectional planes for each technique.
\end{abstract}

MATERIALS AND METHODS: Measurement of both lateral ventricles was performed prospectively in 162 fetuses from 21 to 40 weeks of gestational age referred for evaluation due to increased risk for cerebral pathology. The mean gestational age for evaluation was 32 weeks. The measurements were performed in the customary plane for each technique: axial plane for sonography and coronal plane for MR imaging.

RESULTS: The 2 techniques yielded results in substantial agreement by using intraclass correlation and $\kappa$ coefficient score tests. When we assessed the clinical cutoff of $10 \mathrm{~mm}$, the $\kappa$ score was 0.94 for the narrower ventricle and 0.84 for the wider ventricle, expressing almost perfect agreement. The Bland-Altman plot did not show any trend regarding the actual width of the ventricle, gestational week, or interval between tests. Findings were independent for fetal position, sex, and indication for examination.

CONCLUSIONS: Our study indicates excellent agreement between fetal brain ultrasonography and MR imaging as to the diagnosis of fetal ventriculomegaly in the customarily used sectional planes of each technique.

V

entricular dilation is one of the most common, prenatally diagnosed cerebral abnormalities. 1,2 "Ventriculomegaly" is defined as an atrial diameter exceeding $10 \mathrm{~mm} .^{3,4}$ The prognosis of ventricular dilation depends on the degree of dilation and the presence of associated cerebral or extracerebral abnormalities. ${ }^{5}$ Thus, accurate measurement of the lateral ventricles is of paramount importance in prenatal diagnosis. Recently, guidelines for the assessment of the diameter of the lateral ventricles by using the axial transventricular plane as part of routine fetal sonographic evaluation have been suggested. ${ }^{6,7}$

MR imaging of the fetal CNS is a complementary tool and is performed following detection of abnormalities identified by sonography. The common belief is that fetal CNS MR imaging is

Received August 30, 2013; accepted after revision October 10.

From the Antenatal Diagnostic Unit (S.P., D.S., R.A., E.K.), Department of Obstetrics and Gynecology, and Departments of Diagnostic Imaging (C.H.), and Pediatric Neurology (O.B.Y.), Chaim Sheba Medical Center, Tel HaShomer, Ramat Gan, Israel. (Affiliated with the Sackler School of Medicine, Tel Aviv University, Tel Aviv, Israel.)

Please address correspondence to Sharon Perlman, MD, Antenatal Diagnostic Unit, Department of Obstetrics and Gynecology, Chaim Sheba Medical Center, Tel HaShomer, Ramat Gan, Israel 52621; e-mail: Sharoni5@yahoo.com

http://dx.doi.org/10.3174/ajnr.A3839 more accurate than sonography, particularly when evaluation for associated anomalies is required, when the mother is obese, or when more precise measurement of the ventricular diameter is required. ${ }^{8}$

Measurement of lateral ventricle diameter by using MR imaging is performed on a coronal plane. ${ }^{9}$ Measurement of lateral ventricle diameter by using ultrasound is performed on an axial plane. ${ }^{6}$ This variation may raise possible conflicting classifications due to different sectional planes or use of different tools. The aim of our study was to evaluate the agreement between ultrasonography and MR imaging in the measurement of the lateral ventricle diameter in the customary sectional planes for each technique in prenatal diagnosis.

\section{MATERIALS AND METHODS}

This prospective study was performed in a single tertiary center from January 2011 to December 2012. During this period, 162 singleton fetuses were examined between the ages of 21 and 40 weeks of gestation for increased risk of cerebral pathology. The study was approved by the local institutional ethics committee. Informed consent was obtained from all patients.

The mean and median gestational age when performing MR 
imaging was $32.4 \pm 2.8$ and 32 weeks, respectively (range, 21-40 weeks). The SD and median time lag between ultrasonographic and MR imaging examinations was $6 \pm 14$ days.

The distribution of the number of fetuses as a function of gestational age is represented in Fig 1. Patients were referred to our department for neurosonographic evaluation and cerebral MR imaging following the detection of ventricular dilation or ventricular asymmetry $(n=54,33.3 \%)$ on sonographic examination or suspected cerebral abnormality. Distribution of the number of fetuses as a function of the indication for neurosonographic evaluation is presented in Table 1 . There was no difference in the mean gestational age in regard to the indication.

Fetal presentation was recorded in 147 cases: cephalic in 113 (77\%), breech in $32(21.7 \%)$, and transverse in 2 cases (1.3\%). Fetal sex was recorded in 128 cases: 79 males (62\%) and 49 females (38\%).

Sonographic examinations were performed by using the Voluson E-8 (GE Medical Systems, Zipf, Austria) sonography platforms, equipped with a transabdominal, multifrequency (4- to $8-\mathrm{MHz})$ probe. In our institution, we perform fetal brain MR imaging by using a $1.5 \mathrm{~T}$ system (GE Healthcare Technologies, Milwaukee, Wisconsin). Single-shot fast spin-echo T2-weighted sequences in 3 orthogonal planes were performed by using the following parameters: section thickness, 3-4 mm; no gap; flexible coil (8-channel cardiac coil); matrix, 320/224; TE, 90 ms; and TR, $1298 \mathrm{~ms}$. The FOV was determined by the size of the fetal head: 24 $\mathrm{cm}$ for the smaller fetuses and $\leq 30 \mathrm{~cm}$ for the larger fetuses. T1 fast-spoiled gradient-echo sequences were performed only in the

60

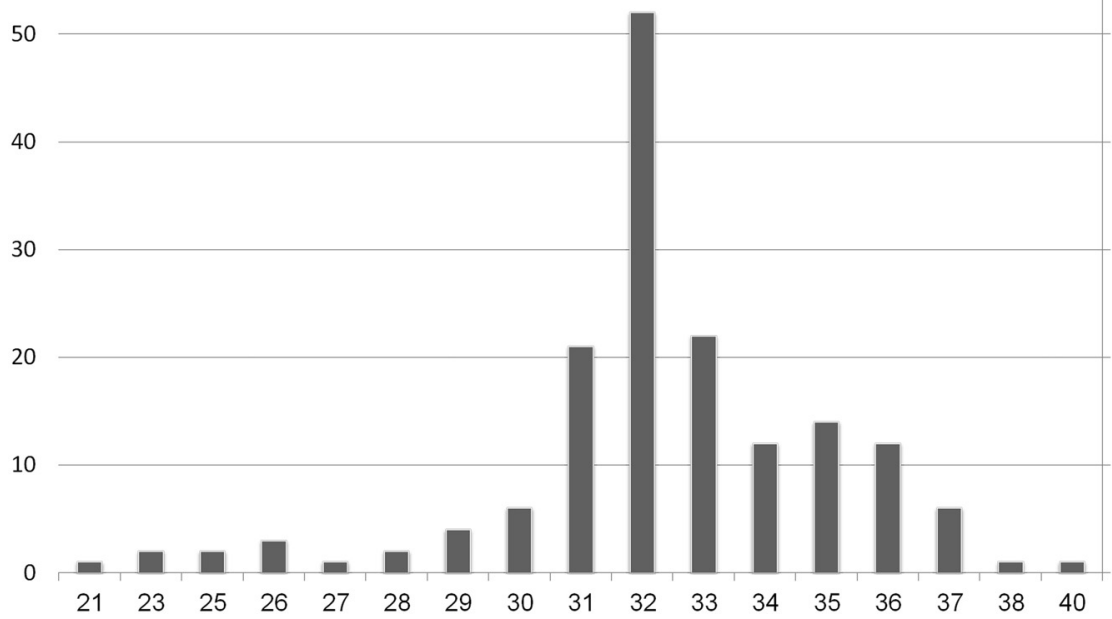

FIG 1. Distribution of number of fetuses providing data as a function of gestational age $(n=162)$. axial plane with a larger FOV (400 mm), with section thickness, 4 mm; gap, $0.5 \mathrm{~mm}$; TR, $160 \mathrm{~ms}$; and TE, $2.3 \mathrm{~ms}$.

For each fetus, both lateral ventricles were measured by ultrasonography and MR imaging by using the same customarily used sectional plane for each technique.

Sonographic measurements were performed in the axial plane as recommended by the International Society of Ultrasound in Obstetrics and Gynecology guidelines for sonographic examination of the fetal central nervous system. ${ }^{6}$ Calipers were positioned inside the echoes generated by the ventricular walls (Fig $2 A$ ). For MR imaging, measurements were obtained on the coronal plane at the level of the ventricles (with good visibility of the choroid plexuses) (Fig 2B). ${ }^{9}$

Sonography is a dynamic, real-time examination, necessitating the presence of the patient; therefore, a single observer performed all measurements. On the other hand, MR imaging results can be accessed as needed. The reproducibility of measurements of the lateral ventricles in MR imaging was assessed as well. Two radiologists (radiologist 1 and radiologist 2) evaluated the entire study sample. Each radiologist was unaware of the results obtained by the other.

Ventricle (a) was defined as narrower and ventricle (b), as wider. We matched the ventricles relatively according to the measurements obtained by MR imaging.

\section{Statistical Analysis}

The width of the measured ventricles in the different planes was compared and statistically analyzed by using the Statistical Package for the Social Sciences software, Version 18.0 (IBM, Armonk, New York).

Quantitative variables are described as median (range), and qualitative variables, as frequency (percentage).

Reproducibility of the sonography and MR imaging measurements of both ventricles was assessed by using BlandAltman analysis and the intraclass correlation coefficient. "Ventriculomegaly" was defined as an atrial diameter exceeding $10 \mathrm{~mm}$ (>4 SDs). The measure of agreement between ultrasonography and MR imaging to define ventriculomegaly was calculated by using the $\kappa$ coefficient. Agreement was considered slight when $\kappa \leq 0.2$; fair when $0.2>\kappa \leq$ 0.4 ; moderate when $0.4<\kappa \leq 0.6$; ex-

Table 1: Distribution of numbers of fetuses providing data as a function of the indication for neurosonography

\begin{tabular}{|c|c|c|c|c|c|}
\hline Indication & No. & Mean Gestational Week & Min & Max & Median \\
\hline CMV seroconversion & 35 & 33.63 & 30 & 40 & 33 \\
\hline Ventricular asymmetry & 54 & 31.78 & 21 & 37 & 32 \\
\hline Abnormal head circumference & 15 & 34.27 & 29 & 38 & 35 \\
\hline Abnormal posterior fossa & 10 & 31.60 & 26 & 35 & 32 \\
\hline Cystic lesions & 8 & 33.75 & 31 & 37 & 33 \\
\hline Family history of CNS illness & 6 & 32.33 & 31 & 34 & 32 \\
\hline Midline abnormalities & 6 & 31.67 & 23 & 37 & 32 \\
\hline Obstetric complications and extra-CNS anatomic findings & 28 & 31.00 & 23 & 36 & 32 \\
\hline
\end{tabular}

Note:-Min indicates minimum; Max, maximum; CMV, cytomegalovirus. 
cellent when $0.6<\kappa \leq 0.8$; and almost-perfect when $\kappa>0.8$.

\section{RESULTS}

The reproducibility of ventricular measurements between sonographic examination and MR imaging was fairly good, as demonstrated by estimates of the mean differences, 95\% limit of agreement, and the intraclass correlation coefficient (Table 2).

There was no particular trend in the Bland-Altman plot for either the narrower (a) or the wider (b) ventricle (Fig 3). When we assessed the clinical cutoff of $10 \mathrm{~mm}$, the $\kappa$ score was 0.94 for axial and coronal planes of the narrower ventricle and 0.84 for the
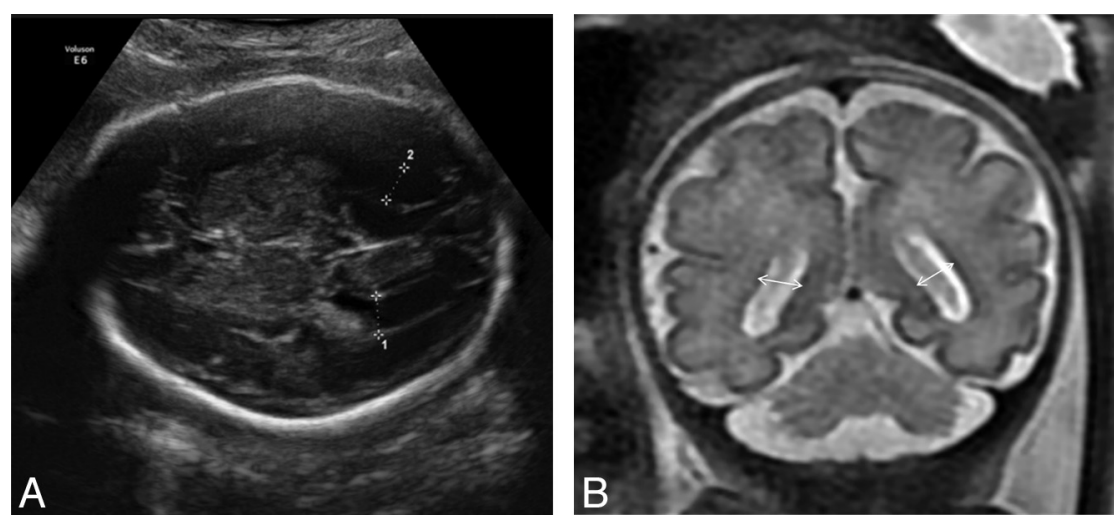

FIG 2. A, Sonographic measurement of the fetal lateral ventricles in the axial transventricular plane. $B$, Measurement of the fetal lateral ventricles in a T2-weighted MR image (coronal plane) at the level of the ventricles; ventricular diameter is measured at the midheight of the ventricle.

Table 2: Reproducibility of measurements of the lateral ventricles for ultrasound and MRI

\begin{tabular}{|c|c|c|c|c|}
\hline & \multicolumn{3}{|c|}{$\begin{array}{l}\text { Differences in Measurement of } \\
\text { Ventricles, Ultrasound-MRI (mm) }\end{array}$} & \multirow{2}{*}{$\begin{array}{c}\text { Definition of } \\
\text { Ventriculomegaly } \\
\kappa \text { Coefficient }\end{array}$} \\
\hline & Mean & $\begin{array}{l}\text { 95\% Limits of } \\
\text { Agreement }\end{array}$ & ICC $(95 \% \mathrm{CI})$ & \\
\hline \multicolumn{5}{|l|}{ MRI radiologist 1} \\
\hline Ventricle (a) & $0.33 \pm 1.58$ & $(-2.76-1.58)$ & $0.91(0.88-0.94)$ & 93.8 \\
\hline Ventricle (b) & $-0.26 \pm 1.58$ & $(-3.35-1.58)$ & $0.94(0.91-0.95)$ & 83.4 \\
\hline \multicolumn{5}{|l|}{ MRI radiologist 2} \\
\hline Ventricle (a) & $-0.88 \pm 1.52$ & $(-3.86-1.52)$ & $0.9(0.78-0.95)$ & 93.8 \\
\hline Ventricle (b) & $-1.09 \pm 1.5$ & $(-4.05-1.5)$ & $0.92(0.75-0.96)$ & 84.2 \\
\hline
\end{tabular}

Note:-ICC indicates intraclass correlation coefficient.
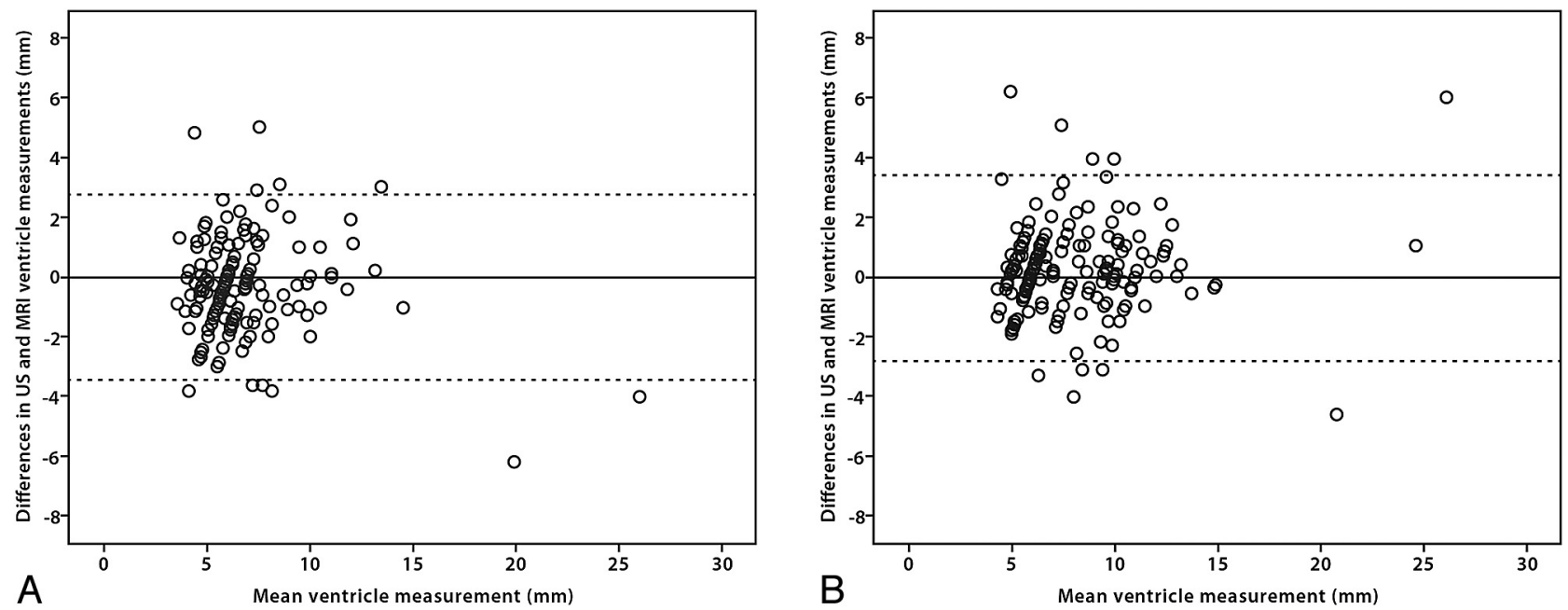

FIG 3. Plots of the difference between sonographic and MR imaging ventricular measurements against their mean (A, narrower ventricle; $B$, wider ventricle). wider ventricle-almost perfect agreement for both evaluating radiologists.

Indeed, when we compared sonographic with MR imaging measurements, 37 cases (radiologist 1) and 35 cases (radiologist 2) of disagreement were observed in the determination of ventriculomegaly (ie, when 1 value was above $10 \mathrm{~mm}$ and the other, below $10 \mathrm{~mm}$ ). In 18 cases, the discrepancy between the 2 measurements was $<1.9 \mathrm{~mm}$. In 15 cases, obesity or previous multiple cesarean deliveries were responsible for poor visibility of the ventricular walls by sonographic examination. In 2 cases, no explanation was found for the disagreement between measurements (Table 3). No specific trend was found when estimating the agreement between measurements in the 2 modalities in re-

When estimating the agreement between sonography and MR imaging measurements as a function of the time interval between tests, the Bland-Altman plot did not indicate any particular trend (Fig 4). Three cases demonstrated great differences between the 2 modalities. In the first, the ventricles were measured as very narrow on MR imaging, most likely below the measurement resolution by sonography $(5 \mathrm{~mm})$. In the second case, the ventricles were very wide $(>20 \mathrm{~mm})$ in both modalities. Both cases represent the extremes of the norm, and measurement differences did not have an impact on clinical decisions. The third case represents a rare case of disagreement between sonography and MR imaging. The time lag between these examinations was a few days; therefore, progressive ventricular dilation could not explain the differences.

The reproducibility of measurements of the lateral ventricles on MR lation to gestational age. 
Table 3: Comparison between ultrasonography and MRI data in the evaluation of ventricular diameter below and above the clinical cutoff of $10 \mathrm{~mm}$

\begin{tabular}{|c|c|c|c|c|}
\hline & \multicolumn{4}{|c|}{ Ultrasonography Axial Plane } \\
\hline & \multicolumn{2}{|c|}{ Ventricle (a) } & \multicolumn{2}{|c|}{ Ventricle (b) } \\
\hline & $<10 \mathrm{~mm}$ & $\geq 10 \mathrm{~mm}$ & $<10 \mathrm{~mm}$ & $\geq 10 \mathrm{~mm}$ \\
\hline \multicolumn{5}{|l|}{$\begin{array}{c}\text { MRI coronal plane, } \\
\text { radiologist } 1\end{array}$} \\
\hline$<10 \mathrm{~mm}$ & 138 & $5^{*}$ & 103 & $14^{*}$ \\
\hline$\geq 10 \mathrm{~mm}$ & $5^{*}$ & 14 & $13^{*}$ & 32 \\
\hline \multicolumn{5}{|l|}{$\begin{array}{l}\text { MRI coronal plane, } \\
\text { radiologist } 2\end{array}$} \\
\hline$<10 \mathrm{~mm}$ & 134 & $1^{* *}$ & 108 & $20^{* *}$ \\
\hline$\geq 10 \mathrm{~mm}$ & $9^{\star \star}$ & 18 & $5^{* *}$ & 25 \\
\hline
\end{tabular}

${ }^{*}$ Cases of disagreement for the diagnosis of ventriculomegaly (ventricular width $\geq 10$ $\mathrm{mm}$ ) between ultrasound and MRI for radiologist 1 , total of 37 cases.

${ }^{* *}$ Cases of disagreement for the diagnosis of ventriculomegaly between ultrasound and MRI for radiologist 2, total of 35 cases.

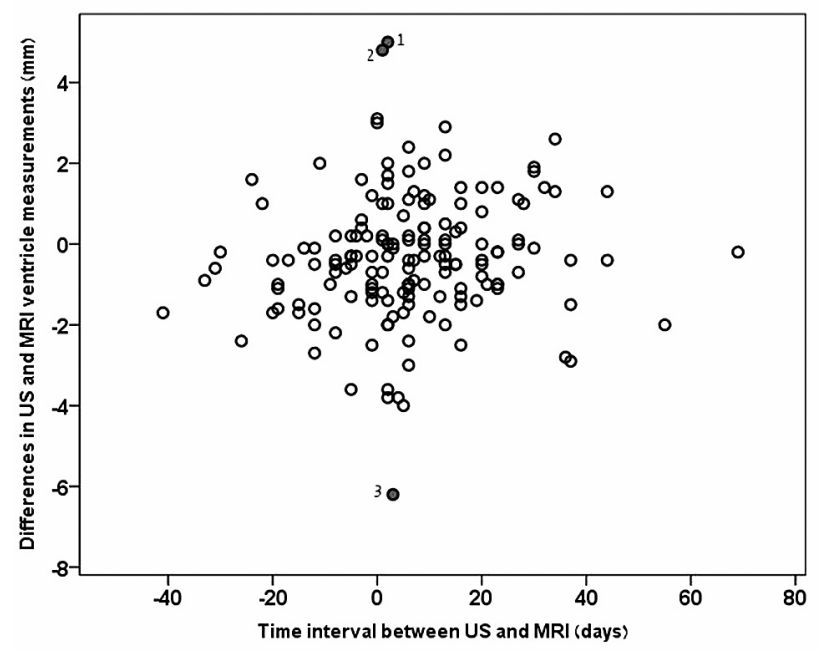

FIG 4. Plots of the difference between sonography and MR imaging ventricular measurements against the time interval between sonography and MR imaging. Cases of great differences between the 2 modalities: 1) very narrow ventricles on MR imaging, most likely below the measurement resolution by sonography $(5 \mathrm{~mm}) ; 2$ ) very wide ventricles $(>20 \mathrm{~mm})$ in both modalities; and 3) a rare case of disagreement between sonography and MR imaging.

Table 4: Interobserver agreement: MRI

\begin{tabular}{lcccc}
\hline & Mean & $\begin{array}{c}\text { 95\% Limits of } \\
\text { Agreement }\end{array}$ & ICC & 95\% Cl \\
\hline Ventricle (a) & $-1.20 \pm 1.11$ & $-3.39-0.97$ & 0.91 & $0.41-0.97$ \\
Ventricle (b) & $-0.82 \pm 1.03$ & $-2.84-1.19$ & 0.96 & $0.84-0.98$ \\
\hline
\end{tabular}

Note:-ICC indicates intraclass correlation coefficient.

imaging was fairly good. The limits of agreement were considered acceptable for clinical purposes, and the intraclass correlation coefficient showed almost perfect agreement (Table 4).

Our findings were independent for fetal sex, presentation, gestational age, and the indication for conducting the neurosonography. The Bland-Altman plot did not indicate any trend regarding the actual width of the ventricle or the time interval between tests.

\section{DISCUSSION}

A great deal of attention has been given to the importance of measuring the fetal lateral ventricular diameter as a pivotal find- ing for prenatal diagnosis of CNS pathologies. ${ }^{1,10,11}$ Ventriculomegaly is one of the most common sonographically detectable fetal cerebral abnormalities. ${ }^{12}$

More than 20 years ago, it was established that the normal fetal ventricular diameter remains relatively constant throughout gestation. ${ }^{10,13}$ Since these reports, $10 \mathrm{~mm}$ is considered the upper limit of the normal range for lateral ventricle diameter measured in the axial plane. The $10-\mathrm{mm}$ cutoff was derived from serial studies that established this cutoff as +3 or +4 SDs, depending on the series. $^{3,14,15}$

The prognosis of ventricular dilation depends on the degree of the dilation and the presence of associated cerebral or extracerebral abnormalities. ${ }^{16,17}$ Thus, accurate measurement of the lateral ventricles is of paramount importance in prenatal diagnosis. Measuring the ventricle diameter reliably is particularly problematic when the ventricular measurement is close to the standard 10 -mm threshold. Measurement of the proximal lateral ventricle is frequently difficult due to near-field artifacts. ${ }^{7}$

It is now accepted that fetal brain MR imaging plays an important role in the prenatal detection of associated cerebral anomalies. ${ }^{8}$ The common understanding is that MR imaging is more accurate than sonographic imaging, particularly in cases of maternal obesity, in identifying or excluding associated anomalies, or in cases in which a more precise measurement of the ventricular diameter is required. ${ }^{18}$

Questions of possible conflicting classifications caused by measurement of the fetal lateral ventricles in different sectional planes by sonography and MR imaging are frequently raised. Usually in sonographic examinations, the fetal ventricle diameter is measured on an axial plane at the level of the thalami as a line perpendicular to the atrium at the level of the posterior margin of the choroid plexus. ${ }^{6}$ Measurement of the lateral ventricle diameter by using MR imaging is performed on a coronal plane. ${ }^{9}$

Good agreement between fetal sonography and MR imaging in the measurement of the lateral ventricle diameter was previously reported when comparing the same coronal planes. ${ }^{19}$ However, the coronal plane is not frequently used as a customary practice in the sonographic examination of the fetal CNS; neither is it mentioned as part of the "fetal neurosonogram" in the International Society of Ultrasound in Obstetrics and Gynecology guidelines. ${ }^{6}$ Others have compared measurements in the axial plane for both imaging modalities, reporting that MR imaging mean ventricular measurements were slightly smaller than sonographic measurements. ${ }^{20}$ However, the axial plane is not commonly used for assessment of the fetal lateral ventricles when using MR imaging.

The aim of our study was to evaluate the agreement between ultrasonography and MR imaging in the measurement of the diameter of the lateral ventricles in the routinely used sectional planes for each technique. Our results indicate that when the fetal lateral ventricles are measured on an axial plane by using ultrasonography and on the coronal plane by using MR imaging, the 2 modalities are in close agreement. Two radiologists assessed the reproducibility of MR imaging measurements of the fetal lateral ventricles in our study. Previous reports have reported moderateto-substantial agreement among MR imaging radiologists in the measurements of the lateral ventricles. ${ }^{9}$ Our study shows high 
agreement between our 2 radiologists in the measurement of both ventricles. Our results showed no influence as to fetal presentation, sex, gestational age, indication for neurosonography, or time lag between tests.

As reported by others, ${ }^{19}$ when the ventricles are very narrow, sonographic evaluation tends to overestimate the ventricular diameter. Our results show that when the fetal ventricular diameter is within normal limits or mildly dilated, ultrasonography and MR imaging are in close agreement. However, in cases of severe ventricular dilation, we found greater discrepancies between sonography and MR imaging. These differences usually do not have significance in clinical decision-making.

MR imaging has a better contrast resolution and is less dependent on maternal body habitus. These features most probably account for most cases with strong disagreement between sonography and MR imaging. This also raises the question as to whether poor visibility of the fetal lateral ventricular walls when conducting prenatal sonography is an indication for prenatal fetal brain MR imaging.

\section{CONCLUSIONS}

Accurate measurement of the lateral ventricles is of paramount importance in prenatal diagnosis, particularly when the ventricular measurement is close to the standard $10-\mathrm{mm}$ threshold of fetal ventriculomegaly. It is now accepted that fetal brain MR imaging plays an important role in the prenatal diagnosis of ventriculomegaly. Possible conflicting classifications caused by measurement of the fetal lateral ventricles in different sectional planes by sonography and MR imaging are frequently found. Our study reveals that in the customarily used sectional planes for sonography and MR imaging, measurements of the fetal lateral ventricles are in close agreement.

\section{REFERENCES}

1. Garel C. Ventricular dilatation. In: Garel C, ed. MRI of the Fetal Brain: Normal Development and Cerebral Pathologies. Berlin: Springer-Verlag; 2004:201-16

2. Leitner $Y$, Goez H, Gull I, et al. Antenatal diagnosis of central nervous system anomalies: can we predict prognosis? J Child Neurol 2004;19:435-38

3. Almog B, Gamzu R, Achiron R, et al. Fetal lateral ventricular width: what should be its upper limit? J Ultrasound Med 2003;22:39-43

4. Salomon LJ, Bernard JP, Ville Y. Reference ranges for fetal ventric- ular width: a non-normal approach. Ultrasound Obstet Gynecol 2007;30:61-66

5. Gaglioti P, Oberto $M$, Todros T. The significance of fetal ventriculomegaly: etiology, short- and long-term outcomes. Prenat Diagn 2009;29:381-88

6. International Society of Ultrasound in Obstetrics \& Gynecology Education Committee. Sonographic examination of the fetal central nervous system: guidelines for performing the 'basic examination' and the 'fetal neurosonogram.' Ultrasound Obstet Gynecol 2007;29:109-16

7. Guibaud L. Fetal cerebral ventricular measurement and ventriculomegaly: time for procedure standardization. Ultrasound Obstet Gynecol 2009;34:127-30

8. Girard N, Ozanne A, Chaumoitre K, et al. MRI and in utero ventriculomegaly. J Radiol 2003;84(12 pt 1):1933-44

9. Tilea B, Alberti C, Adamsbaum C, et al. Cerebral biometry in fetal magnetic resonance imaging: new reference data. Ultrasound Obstet Gynecol 2009;33:173-81

10. Cardoza JD, Goldstein RB, Filly RA. Exclusion of fetal ventriculomegaly with a single measurement: the width of the lateral ventricular atrium. Radiology 1988;169:711-14

11. Filly RA, Cardoza JD, Goldstein RB, et al. Detection of fetal central nervous system anomalies: a practical level of effort for a routine sonogram. Radiology 1989;172:403-08

12. Kelly EN, Allen VM, Seaward G, et al. Mild ventriculomegaly in the fetus, natural history, associated findings and outcome of isolated mild ventriculomegaly: a literature review. Prenat Diagn 2001;21:697-700

13. Siedler DE, Filly RA. Relative growth of the higher fetal brain structures. J Ultrasound Med 1987;6:573-76

14. Farrell TA, Hertzberg BS, Kliewer MA, et al. Fetal lateral ventricles: reassessment of normal values for atrial diameter at US. Radiology 1994;193:409-11

15. Patel MD, Goldstein RB, Tung S, et al. Fetal cerebral ventricular atrium: difference in size according to sex. Radiology 1995; 194:713-15

16. Beeghly M, Ware J, Soul J, et al. Neurodevelopmental outcome of fetuses referred for ventriculomegaly. Ultrasound Obstet Gynecol 2010;35:405-16

17. Melchiorre $\mathrm{K}$, Bhide A, Gika AD, et al. Counseling in isolated mild fetal ventriculomegaly. Ultrasound Obstet Gynecol 2009;34:212-24

18. Browning PD, Laorr A, McGahan JP, et al. Proximal fetal cerebral ventricles: description of ultrasound technique and initial results. Radiology 1994;192:337-41

19. Garel C, Alberti C. Coronal measurement of the fetal lateral ventricles: comparison between ultrasonography and magnetic resonance imaging. Ultrasound Obstet Gynecol 2006;27:23-27

20. Twickler DM, Reichel T, McIntire DD, et al. Fetal central nervous system ventricle and cisterna magna measurements by magnetic resonance imaging. Am J Obstet Gynecol 2002;187:927-31 